\title{
The Importance of Combined Spatio-Temporal Characterization: From in situ to operando Diffraction Measurements of $\mathrm{Li} / \mathrm{Li}_{1.1} \mathrm{~V}_{3} \mathrm{O}_{8}$ Batteries
}

\author{
Esther S. Takeuchi ${ }^{1,2,3}$, Qing Zhang ${ }^{1}$, Andrea M. Bruck ${ }^{2}$, David C. Bock ${ }^{3}$, Kenneth J. Takeuchi ${ }^{1,2}$, \\ Amy C. Marschilok ${ }^{1,2,3}$ \\ ${ }^{1}$ Department of Materials Science and Engineering, Stony Brook University, Stony Brook, NY, USA \\ ${ }^{2}$ Department of Chemistry, Stony Brook University, Stony Brook, NY, USA \\ ${ }^{3}$ Energy Sciences Directorate, Brookhaven National Laboratory, Upton, NY, USA
}

An understanding of electron and $\mathrm{Li}^{+}$transport across multiple size regimes and time scales is critical for understanding phase evolution upon lithiation and delithation of active materials. In this study, $\mathrm{LiV}_{3} \mathrm{O}_{8}$ (LVO) was examined due to its high theoretical capacity of $362 \mathrm{mAh} / \mathrm{g}$ and associated high energy density. The LVO structure can be described as layers of $\mathrm{V}_{3} \mathrm{O}_{8}$ sheets with $\mathrm{Li}^{+}$ions residing in the interlayer space. The parent structure is referred to as alpha $(\alpha)$ phase that transforms to a defected rocksalt structure denoted as beta $(\beta)$ phase upon reaction with $>2.5$ molar equivalents of lithium. Operando angle resolved $\mathrm{x}$-ray diffraction (AXRD) was used to probe the mechanism where the phase evolution was determined as a function of lithiation[1]. Notably, these experiments interrogated the entire cathode sample providing the average phase composition of the electrode as a function of lithiation. Initially, the $\mathrm{Li}_{1.1} \mathrm{~V}_{3} \mathrm{O}_{8}\left(\mathrm{Li}\right.$-poor $\alpha$ ) transforms to $\mathrm{Li}_{2.5} \mathrm{~V}_{3} \mathrm{O}_{8}\left(\mathrm{Li}\right.$-rich $\alpha$ ). Upon further lithiation, the $\mathrm{Li}_{2.5} \mathrm{~V}_{3} \mathrm{O}_{8}$ ( $\mathrm{Li}$-rich $\alpha$ ) phase transforms to $\mathrm{Li}_{4} \mathrm{~V}_{3} \mathrm{O}_{8}(\beta)$ phase. The lithiation mechanism of $\mathrm{LiV}_{3} \mathrm{O}_{8}$ can be described as follows:

(1) $\mathrm{Li}_{1.1} \mathrm{~V}_{3} \mathrm{O}_{8}(\mathrm{Li}$-poor $\alpha)+1.4 \mathrm{e}-\leftrightarrow \mathrm{Li}_{2.5} \mathrm{~V}_{3} \mathrm{O}_{8}(\mathrm{Li}-$ rich $\alpha)$

(2) $\mathrm{Li}_{2.5} \mathrm{~V}_{3} \mathrm{O}_{8}(\mathrm{Li}-$ rich $\alpha)+1.5$ e- $\leftrightarrow \mathrm{Li}_{4} \mathrm{~V}_{3} \mathrm{O}_{8}(\beta)$

(3) $\mathrm{Li}_{4} \mathrm{~V}_{3} \mathrm{O}_{8}(\beta)+1$ e- $\leftrightarrow \mathrm{Li}_{5} \mathrm{~V}_{3} \mathrm{O}_{8}(\beta)$

In bulk electrodes, lithiation may result in a Li ion concentration gradient through the electrode that could vary based on cell and electrode design or electrochemical conditions. Therefore, a technique that allows combined spatio-temporal analysis of phase evolution would enable understanding of the progression of the lithiation reaction, whether the lithiation is limited by ion or electron access, and provide more direct correlation with the electrochemistry. Synchrotron-based characterization using energy dispersive $\mathrm{x}$-ray diffraction (EDXRD) has the capability to penetrate the stainless steel casing of coin cell type batteries allowing both spatial and time-resolved diffraction patterns to be collected without the need for special cell designs or x-ray transparent windows.

Two types of measurements using EDXRD were executed. For both types of experiments, 30 diffraction patterns were collected through the thickness of the cathode beginning at the Li anode interface then progressing to the current collector interface as shown in Figure 1. In situ measurements were conducted such that sealed coin cells were discharged to $0.5,1.5,2.7$, and 3 molar electron equivalents and stored at open circuit voltage for two weeks prior to collection of the EDXRD patterns. Operando experiments were done such that the active cells were discharged while in the beam path and the diffraction patterns were collected while the cells were discharging and charging.

The initial experimental findings for the in situ experiment, showed no variation in phase distribution 
from the Li anode to the current collector interface for any of the cells at any of the discharge levels.[2] The high sensitivity of the beamline would be able to detect structural transformation from $\alpha$ to $\beta$ phase and the presence of non-uniformities, thus, the phase distribution within the electrodes could be assigned as homogeneous. The next set of experiments was conducted under operando conditions where the cells were actively discharged while being interrogated. The operando cells showed heterogeneous phase distributions. The initial lithiation originated at the $\mathrm{Li}$ anode interface and the transformation from Lipoor to Li-rich $\alpha$ phase was observed to propagate toward the current collector region of the electrode. After 2.5 molar electron equivalents of discharge the $\beta$ phase could be visualized, although, initially localized at the Li anode interface. Upon delithiation, the $\beta$ phase could be identified as distributed uniformly across the electrode leading to a homogenous mixture of both phases. After full delithiation $\left(\mathrm{Li}_{1.2} \mathrm{~V}_{3} \mathrm{O}_{8}\right)$ only the Li-poor $\alpha$ phase was detected in the electrode.[3]

These three examples of interrogating the phase progression during lithiation of an electrochemically active electrode highlight the importance of coupled spatial and temporal resolution. Angle resolved operando x-ray diffraction enabled the identification of the phase evolution of the bulk electrode. However, this technique does not provide information relative to the spatial location of the phase transformations. Energy dispersive x-ray diffraction provides spatial resolution of phase formation on lithiation. However, if the cells are predischarged to the phase composition of interest, the phases can relax resulting in a homogeneous composition. Thus, the importance of using operando diffraction measurements that can provide combined spatial and temporal resolution are highlighted. Only that type of experiment provides a complete view of the phase progression within an active battery electrode.

\section{References:}

[1] Q. Zhang et al, Chem. Mater. (2017) 29, p. 2364.

[2] Q. Zhang et al, MRS Advances (2017) p.1.

[3] Q. Zhang et al, Physical Chemistry Chemical Physics (2017) 19, p.14160.

[4] The authors acknowledge funding by the Center for Mesoscale Transport

Properties, an Energy Frontier Research Center supported by the U.S. Department of Energy, Office of Science, Basic Energy Sciences, under Award No. DE-SC0012673.

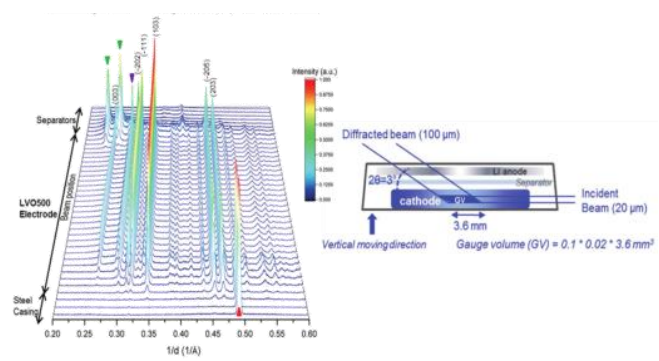

Figure 1. (left) Results of EDXRD experiment showing phase evolution through an electrode, (right) EDXRD experimental setup with respect to the beam position of the coin cell. 